(2) OPEN ACCESS

\begin{abstract}
- Additional material is published online only. To view, please visit the journal online (http://dx.doi.org/10.1136/ oemed-2020-106945).
\end{abstract}

'Department of Social

Epidemiology, Graduate School of Medicine and School of

Public Health, Kyoto University, Kyoto, Japan

2Department of Health Education and Health Sociology, School of Public Health, The University of Tokyo, Tokyo, Japan ${ }^{3}$ Link \& Communication, Tokyo, Japan

${ }^{4}$ Department of Health and Social Behavior, School of Public Health, The University of Tokyo, Tokyo, Japan

\section{Correspondence to}

Koryu Sato, Department of Social Epidemiology, Graduate School of Medicine and School of Public Health, Kyoto University, Kyoto 606-8501, Japan;

sato.koryu.8i@kyoto-u.ac.jp

Received 3 August 2020 Revised 30 December 2020 Accepted 19 January 2021 Published Online First 22 February 2021

\title{
Changes in work and life patterns associated with depressive symptoms during the COVID-19 pandemic: an observational study of health app (CALO
}

\section{mama) users}

\author{
Koryu Sato (1) , ${ }^{1,2}$ Ryohei Sakata, ${ }^{3}$ Chiaki Murayama, ${ }^{3}$ Mai Yamaguchi, ${ }^{2,4}$ \\ Yoko Matsuoka, 2,4 Naoki Kondo 1,2,4
}

\begin{abstract}
Background During the COVID-19 pandemic, many people refrained from going out, started working from home (WFH), and suspended work or lost their jobs. This study examines how such pandemic-related changes in work and life patterns were associated with depressive symptoms.

Methods An online survey among participants who use a health app called CALO mama was conducted from 30 April to 8 May 2020 in Japan. Participants consisted of 2846 users ( 1150 men (mean age $=50.3$ ) and 1696 women (mean age $=43.0)$ ) who were working prior to the government declaration of a state of emergency (7 April 2020). Their daily steps from 1 January to 13 May 2020 recorded by an accelerometer in their mobile devices were linked to their responses. Depressive symptoms were assessed using the Two-Question Screen. Results On average, participants took 1143.8 (95\% $\mathrm{Cl}-1557.3$ to -730.2$)$ fewer weekday steps during the declaration period (from 7 April to 13 May). Depressive symptoms were positively associated with female gender (OR=1.58, 95\% Cl 1.34 to 1.87), decreased weekday steps (OR=1.22, 95\% Cl 1.03 to 1.45$)$ and increased working hours ( $\mathrm{OR}=1.73,95 \% \mathrm{Cl} 1.32$ to 2.26$)$.

Conversely, starting WFH was negatively associated with depressive symptoms $(\mathrm{OR}=0.83,95 \% \mathrm{Cl} 0.69$ to 0.99$)$.

Conclusions Decreased weekday steps during the declaration period were associated with increased odds of depressive symptoms, but WFH may mitigate the risk in the short term. Further studies on the longitudinal effects of WFH on health are needed.
\end{abstract}

\section{INTRODUCTION}

The COVID-19 pandemic is a global threat not only to people's physical health but their mental health as well. ${ }^{1}$ A systematic review including 19 studies from eight countries reported that the prevalence of mental disorders during the pandemic in the general population is relatively high (depression: 14.6\%48.3\%; anxiety: $6.3 \%-50.9 \%$; post-traumatic stress disorder: 7.0\%-53.8\%; psychological distress: 34.4\%-38.0\% ; and stress: $8.1 \%-81.9 \%)$. $^{2}$

In order to prevent the spread of infection, many countries restricted movements of people in various ways, from city quarantine to stay-at-home recommendations. A review exploring evidence during

\section{Key messages}

What is already known about this subject?

- During the COVID-19 pandemic, many people refrained from going out, started working from home, and suspended work or lost their jobs, and these changes in work and life patterns could affect their mental health.

- Previous studies suggest that working from home increases employees' well-being, whereas other studies show that it induces longer working hours and results in the overlapping responsibility of taking care of children by blurring boundaries between work and home time.

- The possible impact of changes in work and life patterns on mental health during the pandemic has only begun to be considered.

What are the new findings?

- Although Japan's antipandemic policy was relatively generous, people's work and life patterns have changed considerably.

- The present study found that decreased weekday steps and increased working hours were associated with increased odds of depressive symptoms during the government declaration of a state of emergency; conversely, starting working from home was negatively associated with risk.

How might this impact on policy or clinical practice in the foreseeable future?

- In the short term, working from home may be recommended to protect employees' mental health; however, employers need to be careful that those who work from home do not work long hours and become physically inactive.

- Health promotions for consistent physical activities, breaking up sedentary time and maintaining social relationships appear to be beneficial to public mental health, especially in the time of a pandemic.

outbreaks of infectious diseases such as severe acute respiratory syndrome and Ebola reported that quarantine had a negative impact on psychological 
outcomes. ${ }^{3}$ Japan's policy during the COVID-19 pandemic was relatively generous compared with other countries that put strict restrictions on going out. The government declared a state of emergency in 7 out of 47 prefectures on 7 April 2020, and was expanded to all prefectures on 16 April. The government asked people to refrain from going out and aimed at a $70 \%$ reduction in the number of people commuting to work. Although this declaration did not include any legally enforceable stipulations, the population largely complied and the number of people going out in major cities saw a $60 \%-80 \%$ reduction. ${ }^{4}$ The number of new COVID-19 cases began decreasing in May, and the declaration was lifted in 39 prefectures on 14 May and in the remaining prefectures on 25 May. The stay-at-home recommendation during the declaration period hampered people from making physical contact with other family members, friends and colleagues, which may have led to feelings of loneliness and depressive symptoms. ${ }^{5} 6$ Moreover, physical inactivity due to staying at home can be associated with depressive symptoms. ${ }^{78}$

The pandemic also had a significant impact on employment and the working environment. Many people suspended work or lost their jobs due to the pandemic, and the sudden changes in employment status could be a risk factor for depressive symptoms. $^{9-11}$ A study showed that those who stopped working due to the pandemic had poorer mental health than those who continued working either at office or from home. ${ }^{12}$ Moreover, the shift to working from home (WFH) could affect employees' mental health in various ways. ${ }^{13}$ WFH may mitigate distress during the pandemic; it can reduce the burden of commuting and help employees maintain social relations with colleagues. ${ }^{13}$ In contrast, some studies suggest that WFH has a tendency to blur boundaries between work and home time, and induces longer working hours ${ }^{14}$ and results in an overlapping responsibility of taking care of children, ${ }^{15}$ which can amplify distress. These possible impacts of changes in the working environment on mental health have only begun to be considered.

In the present study, we examined how pandemic-related changes in work and life patterns were associated with depressive symptoms, using data from a health app called CALO mama. We retrieved longitudinal user data of daily steps and hypothesised that decreased steps in the declaration period represented adherence to the stay-at-home recommendation, which can be associated with depressive symptoms due to physical inactivity and social isolation. We also hypothesised that WFH can be protective against depressive symptoms because it helps workers maintain social relations with their colleagues. This study provides valuable insights on how to prevent depressive symptoms following work and life pattern changes due to the pandemic.

\section{METHODS}

\section{Study participants}

Figure 1 displays a flow chart of our analytical sample. From 30 April to 8 May 2020, we conducted an online survey inviting users of the health app CALO mama provided by Link $\&$ Communication to participate in our study. Users of CALO mama record diet, exercise, mood and quality of sleep every day, and artificial intelligence offers them advice based on their responses. The app also automatically measures the number of daily steps using an accelerometer in the user's mobile device. Out of 7061 respondents, 6392 consented to have their data used for research purposes. Twenty-nine participants answered the questionnaire twice, and we dropped their older responses. There were no missing values in the responses. We excluded 963 participants who were not working prior to the government

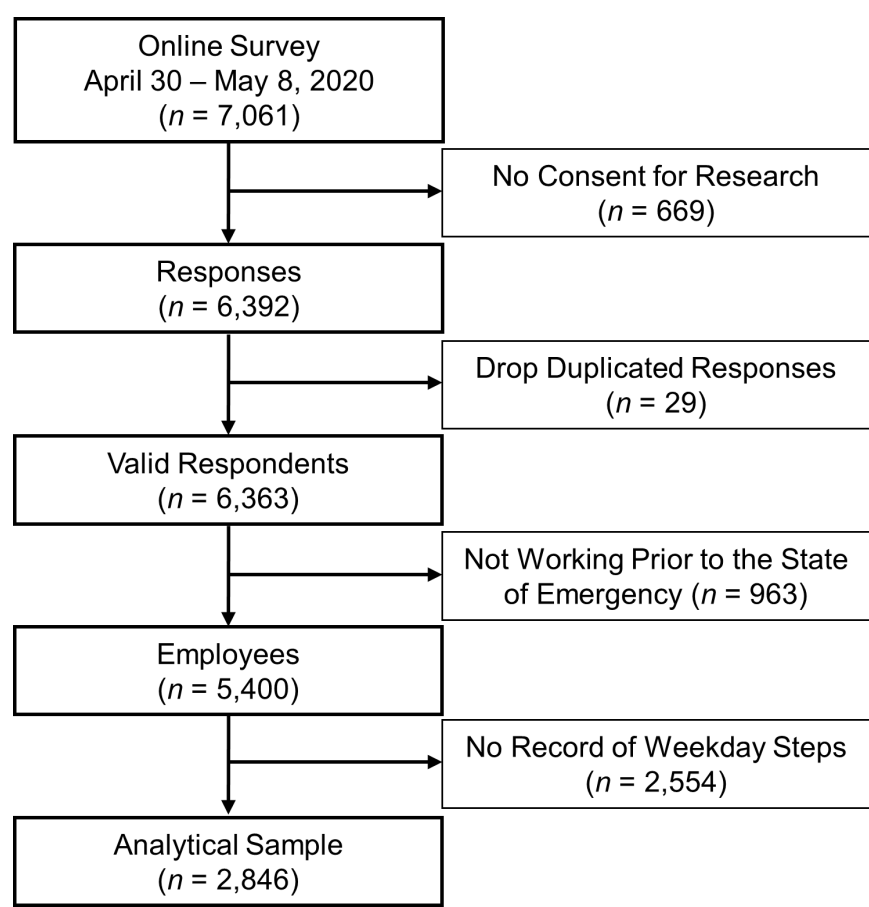

Figure 1 Flow chart of the analytical sample.

declaration of a state of emergency (7 April 2020) from analyses. Out of 5400 participants, 2554 did not record their weekday steps either in the predeclaration period (defined as 1 January-29 February 2020) or the declaration period (defined as 7 April-13 May). Data from the month of March were not used because the number of new cases was increasing in Japan, and thus work and life patterns were shifting in March. Thus, our analytical sample consisted of 2846 participants (1150 men and 1696 women). We compared the characteristics of the analytical sample with those of the excluded sample whose records of weekday steps were not available. We found some differences but confirmed that the prevalence of WFH and depressive symptoms were comparable between the groups (see online supplemental eTable 1).

\section{Survey development}

In the survey, we examined participants' work and life patterns before and after the pandemic by asking 'How did you spend your average weekday before/after the spread of COVID-19?' Participants categorised the average 24-hour day according to time spent working away from home, WFH, commuting, taking care of children, doing housework, exercising, eating, sleeping and other activities, for before and after the declaration (7 April 2020). We defined 'full-time workers' as those whose working hours (both at home and away from home) were 6 or more each day; 'part-time workers' as those whose working hours were less than 6 per day; and 'not working' as those whose working hours were 0 . Changes in work and life patterns were defined as follows: (1) 'decreased weekday steps' represents a binary variable for those whose average weekday steps in the declaration period were fewer than in the predeclaration period; (2) 'suspension of work or loss of employment' accounts for those who had full-time or part-time work in the predeclaration period and were not working in the declaration period; (3) 'increased working hours' refers to those whose working hours increased in the declaration period compared with the predeclaration period; (4) 'shift to WFH' accounts for those who worked more hours outside home in the predeclaration period but worked at home 
more in the declaration period; and (5) 'increased time on childcare' defines those whose time spent taking care of their children increased in the declaration period.

Depressive symptoms were assessed using the Two-Question Screen consisting of the following questions: (1) 'During the past month, have you often been bothered by feeling down, depressed, or hopeless?' and (2) 'During the past month, have you often been bothered by little interest or pleasure in doing things?'16 Those who answered 'Yes' to either or both questions were considered as showing symptoms of depression. Although this two-question screening is simple and short, it has been validated and shows comparable performance with other instruments. 1718

\section{Statistical analysis}

Our analysis is separated into two parts. First, we examined the determinants of weekday steps. The average weekday steps were estimated using a linear mixed model embodying a random effect of individuals. The model has a hierarchical structure with two levels: time variant variables at level 1 were nested into each individual at level 2. Outcome was a continuous variable of average weekday steps; dummy variables indicating the measurement during the declaration period and a full-time worker were variables at level 1; gender and age were measured at level 2. A coefficient of the dummy of the declaration period represented the extent to which the amount of walking decreased due to the state of emergency conditions. We also included interaction terms between the dummy of the declaration period and other covariates in the model. Age was mean-centred for ease of interpretation and avoiding multicollinearity when we added interaction terms.

Second, we used logistic regression to evaluate how changes in work and life patterns were associated with depressive symptoms. In the model, we adjusted for gender, mean-centred age and binary variables indicating those who decreased weekday steps, suspended work or lost a job, increased working hours, started WFH and increased time on childcare. All analyses were performed using Stata V.16.1.

\section{RESULTS}

Table 1 delineates the characteristics of the participants. The number of full-time workers decreased in the declaration period for both men and women. About 70\% of participants decreased their weekday steps. Men were more likely to shift to WFH (32.3\% of men compared with $23.5 \%$ of women). More women experienced suspension of work or loss of employment than men (14.0\% of women compared with $3.7 \%$ of men). Depressive symptoms were more prevalent among women $(45.1 \%$ of women compared with $32.6 \%$ of men).

Figure 2 depicts the trends in the average number of daily steps in year 2020. For both men and women, the average steps in the declaration period were fewer than in the predeclaration period.

Table 2 presents the results from a mixed-effect model estimating the association of average weekday steps with the declaration and with other participant characteristics. The declaration period was associated with decreased walking (coefficient $=-1143.8,95 \%$ CI -1557.3 to -730.2 ). Interaction terms with a dummy variable of the declaration period suggested that male and younger participants were more likely to see a decrease in the number of weekday steps during the declaration period.

Table 3 shows the association between depressive symptoms and changes in work and life patterns. Depressive symptoms were positively associated with female gender $(\mathrm{OR}=1.58$, $95 \%$ CI 1.34 to 1.87$)$, decreased weekday steps $(\mathrm{OR}=1.22$, $95 \%$ CI 1.03 to 1.45$)$ and increased working hours $(\mathrm{OR}=1.73$, 95\% CI 1.32 to 2.26 ). Conversely, starting WFH was negatively associated with depressive symptoms (OR $=0.83$, 95\% CI 0.69 to 0.99 ). To consider regional heterogeneity, we added a dummy

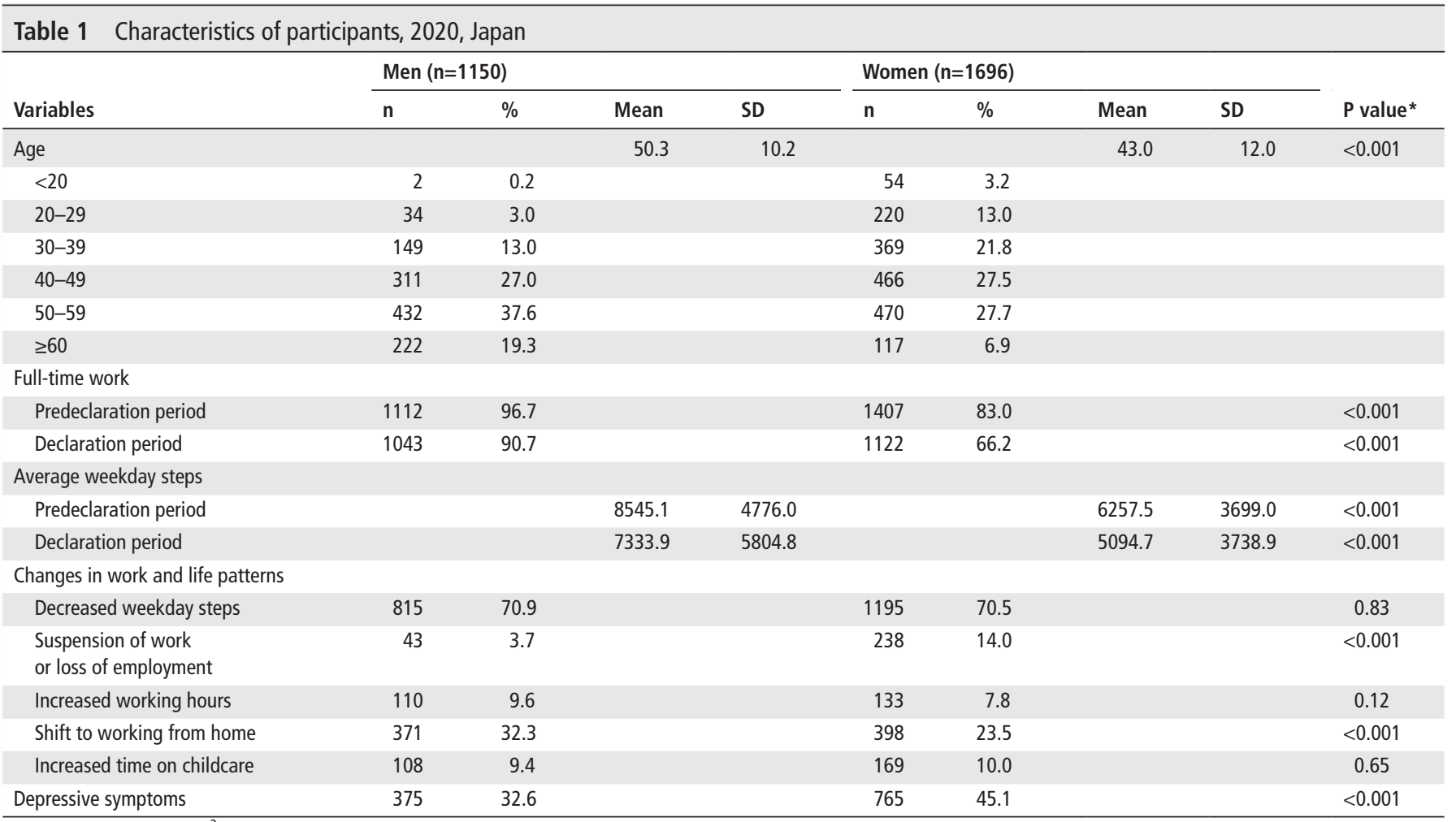

${ }^{*} \mathrm{P}$ values of t-test and $\chi^{2}$ test for gender differences. 


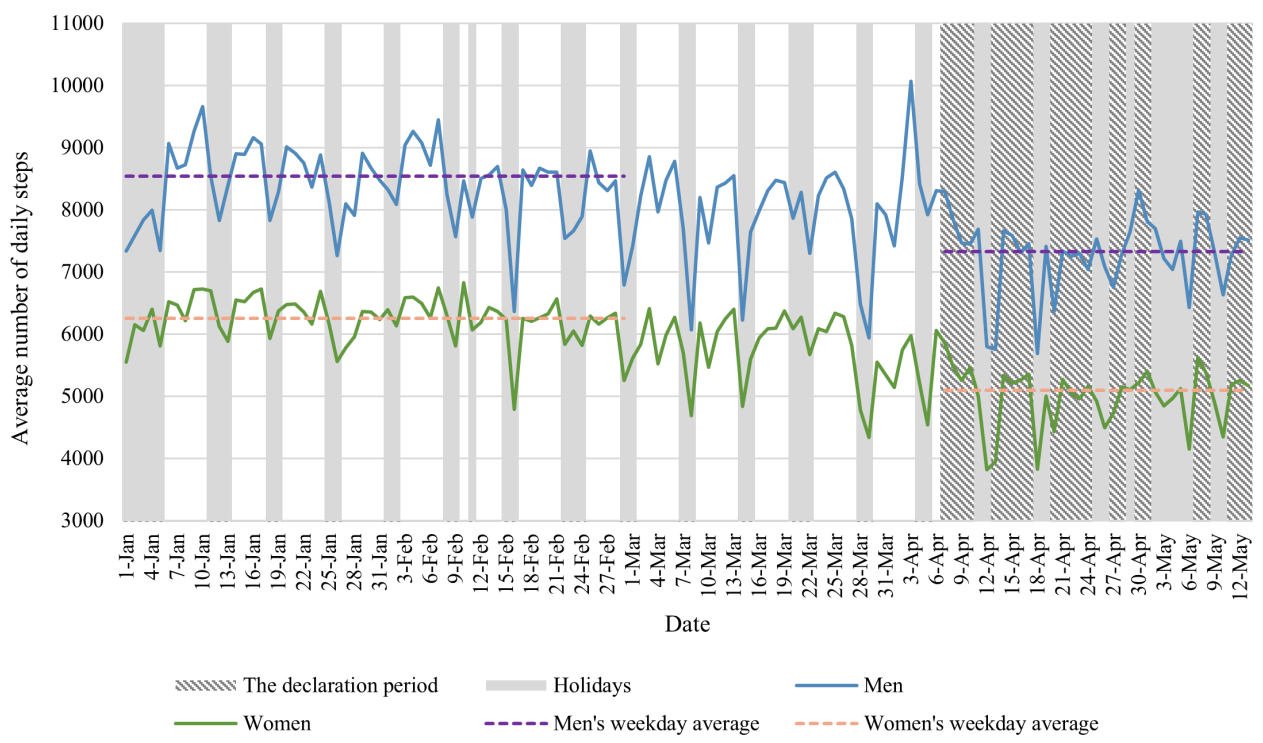

Figure 2 Trends in the average number of daily steps by gender, 2020, Japan. Data from March were not used in the analyses because new cases of COVID-19 were increasing in Japan at the time and thus lifestyles were shifting.

variable for 13 prefectures placed on a special alert when the local governments recognised that COVID-19 was spreading very rapidly (information on prefecture of residence was available for 2216 out of 2846 participants). The dummy variable was not associated with depressive symptoms, and we obtained similar results to those presented above. In addition, we also examined a binary variable indicating increased time on exercise during the declaration period. The variable appeared not to be associated with depressive symptoms and thus we did not include it in the model.

\section{DISCUSSION}

The present study investigated how pandemic-related changes in work and life patterns were associated with depressive symptoms, using data from CALO mama users. Studying the significant changes resulting in psychological consequences during the COVID-19 pandemic is important because the measures against the spread of infection including stay-at-home recommendations have inevitably modified work and life patterns in the large proportion of the population. ${ }^{19}$ On average, participants saw a decrease of more than 1100 weekday steps during the declaration period. Depressive symptoms were positively associated with decreased weekday steps and increased working

Table 2 Association of average weekday steps with the declaration and participants' characteristics, 2020, Japan*

\begin{tabular}{lcc}
\hline Variable & Coefficient & \multicolumn{1}{l}{$95 \% \mathrm{Cl}$} \\
\hline Declaration period & -1143.8 & -1557.3 to -730.2 \\
\hline Women & -2198.2 & -2551.0 to -1845.3 \\
Women $\times$ declaration period & 266.6 & 2.5 to 530.7 \\
Age & -0.1 & -14.5 to 14.4 \\
Age $\times$ declaration period & 25.3 & 10.9 to 39.6 \\
Full-time work & 654.8 & 264.2 to 1045.3 \\
Full-time work $\times$ declaration period & -152.5 & -533.5 to 228.4 \\
Constant & 7912.3 & 7448.0 to 8376.6
\end{tabular}

*The model is adjusted for gender, mean-centred age, a dummy variable indicating a full-time worker, and a dummy variable indicating the measurement of the declaration period and its interaction terms with other covariates. The coefficient represents the number of average weekday steps. hours. Conversely, starting WFH was negatively associated with depressive symptoms.

Given decreased weekday steps represented physical inactivity during the declaration period, our findings are consistent with previous studies which showed associations between physical inactivity and increased risks of depression during the COVID-19 pandemic. ${ }^{21}{ }^{21}$ Physical activity can be protective against depression through biological and psychosocial pathways, ${ }^{7}$ but the stay-at-home recommendation prevented people from engaging in physical activity and may have elevated the risk of depression. In terms of biological mechanism, it has been suggested that physical activity can enhance neuroplasticity and reduce inflammatory, oxidative stress and cortisol release from the hypothalamic-pituitary-adrenal (HPA) axis. ${ }^{7}$ These functions of physical activity can prevent damages to the brain and reductions in the volume of hippocampal, prefrontal and anterior cingulate cortex, which are associated with depression. ${ }^{22-24}$ In terms of psychosocial mechanism, physical activity is associated with positive perceptions such as self-esteem (defined as 'a global evaluation of self-worth and self-image, encompassing cognitive, behavioral and affective processes') and self-efficacy (defined as 'the belief that one can achieve a specific task'), which are protective against depression. ${ }^{7}$ The stay-at-home recommendation may have interfered with opportunities to gain such positive perceptions through exercise. Moreover, people who were physically active in the predeclaration period may be more likely

Table 3 OR for depressive symptoms during the declaration period, 2020, Japan

\begin{tabular}{lll}
\hline Variables & OR & $95 \% \mathrm{Cl}$ \\
\hline Women & 1.58 & 1.34 to 1.87 \\
\hline Age* $^{*}$ & 0.99 & 0.98 to 0.998 \\
Decreased weekday steps & 1.22 & 1.03 to 1.45 \\
Suspension of work or loss of employment & 1.01 & 0.78 to 1.32 \\
Increased working hours & 1.73 & 1.32 to 2.26 \\
Shift to working from home & 0.83 & 0.69 to 0.99 \\
Increased time on childcare & 1.08 & 0.83 to 1.40 \\
Constant & 0.43 & 0.36 to 0.52 \\
\hline
\end{tabular}

${ }^{*}$ Mean-centred age. 
to be frustrated by restrictions in daily activity during the declaration period than those who were inactive. Interestingly, a study examining Chinese adults found a negative association between time spent on exercise and life satisfaction during the COVID-19 pandemic. ${ }^{12}$ Another COVID-19 study in Brazil showed that those who became physically inactive during the pandemic presented higher odds of loneliness, whereas those who were consistently inactive did not show a clear correlation with it, compared with those who were consistently active. ${ }^{21}$ People who were consistently inactive may have been able to adapt to the stay-at-home habits easily, whereas those who had been active might have had difficulty in coping with the discrepancy.

In terms of social interactions, we assumed that decreased weekday steps also represented social isolation (defined as 'inadequate quality and quantity of social relations with other people at the individual, group, community, and larger social environment levels where human interaction takes place ${ }^{25}$ ) during the declaration period. According to a survey in Japan, $66.3 \%$ of people answered that they had fewer opportunities to meet people face-to-face in communication during the declaration period than they did a year ago. ${ }^{26}$ Social isolation can evoke feelings of loneliness, and stress from loneliness activates the HPA axis more frequently and elevates cortisol level, which can contribute to the pathophysiology of depression. ${ }^{6}$ Our findings are in line with other COVID-19 studies on approximate concepts, which found that low levels of social capital ('a series of resources that individuals earn as a result of their membership in social networks ${ }^{25}$ ) and social support (consisting of 'functional social support, the functions fulfilled by social relations, and structural social support, the existence, quantity, and properties of social relations ${ }^{25}$ ) were associated with increased anxiety and stress. ${ }^{27} 28$

In the present study, starting WFH was negatively associated with depressive symptoms. This is consistent with previous studies which have shown that WFH increases employees' well-being. ${ }^{29}{ }^{30}$ In the context of COVID-19, WFH may have been beneficial to mental health because it enabled workers to keep working and communicating with their colleagues during the declaration period, which helped them have a sense of belonging. WFH may also reduce the fear of infection at or on the way to work. A study at the early stage of the pandemic showed that the number of workplace measures for COVID19 , including encouraging employees to start WFH, was negatively correlated with employees' psychological distress. ${ }^{31}$ In Japan, WFH had not taken root for a long time; only $8.4 \%$ of employees had worked from home as of September 2019. ${ }^{32}$ However, WFH spread rapidly due to the widespread restrictions in movement. A national survey reported that $34.6 \%$ of employees worked from home during the pandemic, ${ }^{33}$ while $30.0 \%$ of men and $19.1 \%$ of women in the present study shifted their primary workplace to their homes. WFH can reduce the burden of commuting. ${ }^{13}$ Meanwhile, some who started WFH may increase time on exercise or other activities, but others may blur boundaries between work and home time and increase working hours. ${ }^{14}$ One study reported that those who started WFH during the COVID-19 pandemic increased sedentary and screen time. ${ }^{34}$ Our results showed that increased working hours were associated with depressive symptoms, which could offset the benefits from WFH. Moreover, the negative consequences of sedentary behaviours on health are well known..$^{35}$ Further studies on the longitudinal effects of WFH on mental and physical outcomes are needed.

The present study has several limitations. First, it lacks generalisability because participants were recruited from a health app. We compared the characteristics of our participants with those of a national survey in online supplemental eTable 2. Our analytical sample included more women, persons aged 40-59 years and full-time workers, but less persons aged 20-29 years and 60 or older, than the national survey of employed persons. Male participants walked more than the national average in the pre-declaration period, whereas female participants walked less. Fewer male participants experienced suspension of work or loss of employment during the declaration period than the national average, whereas more female participants experienced it. Moreover, participants may be health-conscious enough to manage their daily health activities through the app, and such unobserved factors may cause selection bias. Thus, a populationbased study is needed in the future. Second, although the number of daily steps was measured longitudinally, participants responded by recalling how they spent their time before the pandemic. Our questionnaire at the time of the study was not formally validated, although our measure of depressive symptoms has been validated and widely used. ${ }^{17}{ }^{18}$ Hence, there is a possibility of recall bias. Third, one cannot conclude a causal relationship from this study because we could not assess mental health conditions prior to the pandemic. If a significant number of participants suffered from depressive symptoms prior to the pandemic, this would alter the interpretation of the findings. Fourth, we could not adjust for some important covariates such as education, household income, number of children, marital status and physical health history due to space constraints in the survey. If these factors were confounded, the estimations could be biased.

In conclusion, the study shows that weekday steps decreased during the declaration period and that a decrease in walking was associated with depressive symptoms. WFH may mitigate the risk, but at the same time may induce longer working hours. Our findings show that increased working hours during the declaration period were associated with depressive symptoms. Employers and the regulatory authority should monitor employees' working hours and ensure minimum rest periods to protect employees' health. Health promotions for consistent physical activities, breaking up sedentary time and maintaining social relationships during the pandemic appear to be beneficial to public mental health. For individuals, it is important to keep track of health behaviours to help them monitor changes in work and life patterns and compensate for potentially detrimental factors.

Contributors KS conceived the study design, performed the analysis and drafted the manuscript. RS and CM collected the data. All authors developed the questionnaire, interpreted the results and revised the manuscript critically.

Funding This study was supported by the Grants-in-Aid for Scientific Research (KAKENHI) Program (grant 20K18931) from the Japan Society for the Promotion of Science. The funder of the study had no role in study design, data collection, analysis, interpretation, writing of the article or the decision to submit for publication.

\section{Competing interests None declared.}

\section{Patient consent for publication Not required.}

Ethics approval This study was approved by the Research Ethics Committee of Graduate School of Medicine and Faculty of Medicine, the University of Tokyo (2019372NI-(1)).

Provenance and peer review Not commissioned; externally peer reviewed.

Data availability statement Data are available upon reasonable request to Link \& Communication.

Supplemental material This content has been supplied by the author(s). It has not been vetted by BMJ Publishing Group Limited (BMJ) and may not have been peer-reviewed. Any opinions or recommendations discussed are solely those of the author(s) and are not endorsed by BMJ. BMJ disclaims all liability and responsibility arising from any reliance placed on the content. Where the content 
includes any translated material, BMJ does not warrant the accuracy and reliability of the translations (including but not limited to local regulations, clinical guidelines, terminology, drug names and drug dosages), and is not responsible for any error and/or omissions arising from translation and adaptation or otherwise.

Open access This is an open access article distributed in accordance with the Creative Commons Attribution Non Commercial (CC BY-NC 4.0) license, which permits others to distribute, remix, adapt, build upon this work non-commercially, and license their derivative works on different terms, provided the original work is properly cited, appropriate credit is given, any changes made indicated, and the use is non-commercial. See: http://creativecommons.org/licenses/by-nc/4.0/.

\section{ORCID iD}

Koryu Sato http://orcid.org/0000-0002-8418-8535

\section{REFERENCES}

1 Bao Y, Sun Y, Meng S, et al. 2019-nCoV epidemic: address mental health care to empower society. The Lancet 2020;395:e37-8.

2 Xiong J, Lipsitz O, Nasri F, et al. Impact of COVID-19 pandemic on mental health in the general population: a systematic review. J Affect Disord 2020;277:55-64.

3 Brooks SK, Webster RK, Smith LE, et al. The psychological impact of quarantine and how to reduce it: rapid review of the evidence. The Lancet 2020;395:912-20.

4 Agoop Corp. Analysis of human flow changes in the spread of a novel coronavirus, 2020. Available: https://corporate-web.agoop.net/pdf/covid-19/agoop_analysis_ coronavirus.pdf [Accessed 12 Jun 2020].

5 Kawachi I, Berkman LF. Social ties and mental health. J Urban Health 2001;78:458-67.

6 Cacioppo JT, Cacioppo S, Capitanio JP, et al. The Neuroendocrinology of social isolation. Annu Rev Psychol 2015;66:733-67.

7 Kandola A, Ashdown-Franks G, Hendrikse J, et al. Physical activity and depression: towards understanding the antidepressant mechanisms of physical activity. Neurosci Biobehav Rev 2019:107:525-39.

8 Schuch FB, Vancampfort D, Firth J, et al. Physical activity and incident depression: a meta-analysis of prospective cohort studies. Am J Psychiatry 2018;175:631-48.

9 Yoo K-B, Park E-C, Jang S-Y, et al. Association between employment status change and depression in Korean adults. BMJ Open 2016;6:e008570.

10 Virtanen $\mathrm{P}$, Janlert U, Hammarström A. Exposure to temporary employment and job insecurity: a longitudinal study of the health effects. Occup Environ Med 2011;68:570-4.

11 Burgard SA, Brand JE, House JS. Toward a better estimation of the effect of job loss on health. J Health Soc Behav 2007;48:369-84.

12 Zhang SX, Wang Y, Rauch A, et al. Unprecedented disruption of lives and work: health, distress and life satisfaction of working adults in China one month into the COVID-19 outbreak. Psychiatry Res 2020;288:112958.

13 Bouziri H, Smith DRM, Descatha A, et al. Working from home in the time of COVID-19: how to best preserve occupational health? Occup Environ Med 2020;77:509-10.

14 Eurofound and the International Labour Office. Working Anytime, anywhere: the effects on the world of work. Geneva Publications Office of the European Union, Luxembourg, and the International Labour Office; 2017. http://eurofound.link/ef1658 [Accessed 22 Jun 2020].

15 Standen P, Daniels K, Lamond D. The home as a workplace: Work-family interaction and psychological well-being in telework. J Occup Health Psychol 1999:4:368-81.

16 Spitzer RL, Williams JB, Kroenke K, et al. Utility of a new procedure for diagnosing mental disorders in primary care. The PRIME-MD 1000 study. JAMA 1994:272:1749-56.
17 Whooley MA, Avins AL, Miranda J, et al. Case-Finding instruments for depression. two questions are as good as many. J Gen Intern Med 1997;12:439-45.

18 Tsoi KKF, Chan JYC, Hirai HW, et al. Comparison of diagnostic performance of TwoQuestion Screen and 15 depression screening instruments for older adults: systematic review and meta-analysis. Br J Psychiatry 2017;210:255-60.

19 Arora P, Desai K. Impact of Affordable Care Act coverage expansion on women's reproductive preventive services in the United States. Prev Med 2016;89:224-9.

20 Schuch FB, Bulzing RA, Meyer J, et al. Associations of moderate to vigorous physical activity and sedentary behavior with depressive and anxiety symptoms in self-isolating people during the COVID-19 pandemic: a cross-sectional survey in Brazil. Psychiatry Res 2020;292:113339.

21 Werneck AO, Silva DR, Malta DC, et al. Physical inactivity and elevated TV-viewing reported changes during the COVID-19 pandemic are associated with mental health: a survey with 43,995 Brazilian adults. J Psychosom Res 2021;140:110292.

22 Schmaal L, Veltman DJ, van Erp TGM, et al. Subcortical brain alterations in major depressive disorder: findings from the ENIGMA major depressive disorder working group. Mol Psychiatry 2016;21:806-12.

23 Bora E, Fornito A, Pantelis C, et al. Gray matter abnormalities in major depressive disorder: a meta-analysis of voxel based morphometry studies. J Affect Disord 2012;138:9-18.

24 Zhao Y-J, Du M-Y, Huang X-Q, et al. Brain grey matter abnormalities in medicationfree patients with major depressive disorder: a meta-analysis. Psychol Med 2014:44:2927-37.

25 Wang J, Lloyd-Evans B, Giacco D, et al. Social isolation in mental health: a conceptual and methodological review. Soc Psychiatry Psychiatr Epidemiol 2017:52:1451-61.

26 Nippon Research Center Ltd. Survey on Communication [Part1] - Fewer Opportunities for Meeting People and Increased Online Communication, 2020. Available: https:// www.nrc.co.jp/report/200825.html [Accessed 17 Dec 2020].

27 Xiao H, Zhang Y, Kong D, et al. Social capital and sleep quality in individuals who Self-Isolated for 14 days during the coronavirus disease 2019 (COVID-19) outbreak in January 2020 in China. Med Sci Monit 2020;26:e923921.

28 Cao W, Fang Z, Hou G, et al. The psychological impact of the COVID-19 epidemic on college students in China. Psychiatry Res 2020;287:112934.

29 Bloom N, Liang J, Roberts J, et al. Does working from home work? Evidence from a Chinese experiment *. Q J Econ 2015;130:165-218.

30 Kazekami S. Mechanisms to improve labor productivity by performing telework. Telecomm Policy 2020;44:101868.

31 Sasaki N, Kuroda R, Tsuno K, et al. Workplace responses to COVID-19 associated with mental health and work performance of employees in Japan. J Occup Health 2020:62:e12134.

32 Ministry of Internal Affairs and Communications. Communication usage survey 2019, 2020. Available: https://www.soumu.go.jp/johotsusintokei/statistics/data/200529_1. pdf [Accessed 22 Jun 2020].

33 Cabinet Office. A survey on changes in lifestyle awareness and behavior under the influence of a new coronavirus infection, 2020. Available: https://www5.cao.go.jp/ keizai2/manzoku/pdf/shiryo2.pdf [Accessed 22 Jun 2020].

$34 \mathrm{McD}$ owell CP, Herring MP, Lansing J, et al. Working from home and job loss due to the COVID-19 pandemic are associated with greater time in sedentary behaviors. Front Public Health 2020;8:597619.

35 Young DR, Hivert M-F, Alhassan S, et al. Sedentary behavior and cardiovascular morbidity and mortality: a science Advisory from the American heart association. Circulation 2016;134:e262-79. 